\title{
Micro-naciones del sí-mismo en tiempos de guerra: análisis de discurso y psicología
}

\author{
Micro-Nations of the Self in Times of War: \\ Discourse Analysis and Psychology
}

Recibido: noviembre 14 de 2011 | Revisado: mayo 10 de 2012 | Aceptado: junio 10 de 2012

\author{
IAN PARKER * \\ Discourse Unit, Manchester, Reino Unido
}

\section{RES UMEN}

Este artículo considera problemas del análisis de discurso contemporáneo referidos a los siguientes tópicos: su foco en la conversación cotidiana, las secuencias formales, la explicación correcta y la segregación disciplinaria. Problemas que replican dificultades generales que muestra la disciplina de la psicología, tales como la mirada puesta sobre aquellos que están fuera de su dominio, el reduccionismo a nivel del individuo, la abstracción de la conducta y los procesos cognitivos, los reclamos a la autoridad interpretativa y la evitación de la política como tal. Se describen aproximaciones a la práctica discursiva desde dentro de una de las recién formadas micronaciones, la cual, a su vez, provee una nueva manera de pensar el papel de la psicología en procesos sociales más amplios. Los siguientes principios alternativos para la práctica discursiva se derivan de esta descripción: dirigir la mirada de nuevo sobre la psicología y las fuerzas ideológicas que le dieron cabida; tratar las formas de representación como puntos para el ejercer el poder; analizar las formas sociales de manera situada contextual e históricamente; destacar formas de práctica discursiva que abren espacios para la argumentación acerca de la naturaleza de la interpretación y conectar las contradictorias fuerzas afectivas individuales con la lucha política. Palabras clave autor

análisis de discurso, psicología crítica, practica discursiva, nación, sí-mismo.

Palabras clave descriptores

Psicología social, Psicología política.

\section{A B S T R A C T}

This paper outlines problems with contemporary discourse analysis - a focus on everyday conversation, interpersonal interaction, formal sequences, correct explication and disciplinary segregation - problems which replicate general problems with the discipline of psychology (the gaze upon those outside its domain, reduction to the level of the individual, abstraction of behaviour and cognitive processes, claims to interpretative authority, and avoidance of politics as such). Approaches to discursive practice are described from within one of the recently-formed micro-nations which also provide a new way of thinking about the role of psychology in broader social processes. Alternative principles for discursive practice are derived from this description: turning the gaze back onto psychology and the ideological forces that give rise to it; treating forms of representation as sites for the relay of power; tracing how social forms are treated as contextually and historically situated; highlighting forms of discursive practice that open up spaces for interpretation and argument about the nature of interpretation; and connecting contradictory individual affective forces with the realm of political struggle.

Key words author

Discourse analysis, critical Psychology, discursive practice, nation, self.

Key words plus

Social Psychology, Politic Psychology. 


\section{Introduciendo la psicología}

Se ha reportado que la reina de Inglaterra le confió a un mayordomo los misterios que rodean la muerte de Diana, la Princesa de Gales, diciendo que "hay fuerzas oscuras obrando, de las que no sabemos nada" (Blackburn, 2005, pp. 25-26).

Este comentario no estaría fuera de lugar en tiempos medievales, en ese entonces había una relativa clara demarcación entre las fuerzas del bien y el mal. A esas fuerzas se les entendería como algo externo que podría tentar y convertir a los individuos. Bajo el capitalismo, sin embargo, ocurre un cambio de foco de manera tal que al mismo tiempo hay todavía una creencia en conspiraciones oscuras, de las cuales nada o casi nada sabemos, y hay también la búsqueda de fuerzas oscuras dentro de los individuos, de las cuales nada o casi nada sabemos. La idea de que no sabemos casi nada de estas fuerzas oscuras nos lleva al borde del conocimiento y la razón, y también nos conduce a definir la psicología humana como un lugar de investigación que contiene misterios.

La referencia de la reina a 'fuerzas oscuras' también dirige la atención a la permanente preocupación por la pureza del Estado nación, el cual es amenazado por la negra oscuridad y la confusión, cuestiones que invocan en la imaginación occidental el mundo caótico y salvaje externo a Europa, quizás más precisamente en este caso, el mundo fuera del territorio inglés (Achcar, 2006).

Con la llegada del capitalismo, la estructura metafórica de la comunidad civilizada se separa en dos aspectos idénticos que se complementan entre sí: la nación y el sí-mismo. La nación se concibe como un cuerpo político y el sí-mismo viene a ser visto como si fuera una nación microscópica. Entonces, la disciplina psicológica viene a operar como aparato de Estado, definiendo y regulando la buena conducta y a la vez comprometida con proponer unas formas del cuerpo como análogas a territorios geográficos. Esta es la conexión rechazada entre la nación y el sí-mismo y entre la disciplina y el Estado. Relación que le da el carácter ideológico al sujeto de la psicología, tanto a nivel del sujeto individual como del sujeto disciplinario (Parker, 2010).
En el tiempo de desregulación neoliberal de los servicios del estado de bienestar y de individualización intensificada, que es, también, un tiempo de vigilancia y concentración del poder en los antiguos centros imperialistas, ciertamente es como si estuviéramos en una guerra gobernada por fuerzas de las que sabemos casi nada. Hay al menos tres niveles en esta guerra que están entretejidos. El primer nivel es por el territorio geográfico, el cual muy a menudo procede de una manera virtualmente mediada -tanto para los combatientes como para la audiencia global-, al punto que parece apropiado decir que la guerra ocurre y no ocurre al mismo tiempo (Baudrillard, 1995). En el segundo nivel la connivencia de los psicólogos con la guerra empuja, por ejemplo, a la Asociación Americana de Psicología (APA) a legitimar el uso de la tortura sobre aquellos que son, de ese modo, sometidos a una clase de vida inhumana "vida nuda" (Agamben, 1998). Y hay un tercer nivel de guerra, uno que parece trivial en comparación con los otros dos niveles, excepto que todavía es para muchos de aquellos que pelean por una cierta clase de racionalidad en la investigación académica y clínica, una guerra sobre los paradigmas científicos dentro de la psicología; aquí es como si cada lado le insistiera al otro que "no va a ganar esta guerra, no puede ganar esta guerra" (Parker, 2010).

\section{Análisis de discurso}

Esto me conduce a la disputa, aparentemente más trivial en la psicología, sobre el papel del análisis del discurso. El análisis de discurso en la disciplina de la psicología fue, desde un comienzo, una contienda sobre el lenguaje y las definiciones del lenguaje. Parecía, en cierto punto, como si el centro del argumento acerca del discurso en la psicología fuese sobre su misma existencia como una disciplina independiente (Parker, 2002, 2004). Mi argumento ahora es que aquellos de nosotros que hicimos un giro hacia el discurso en la psicología cometimos un error necesario, es decir, argumentamos en términos que no pudimos escoger, pero ahora es necesario reconocer que no podemos ganar esta guerra en aquellos términos. Debemos encontrar 
un lugar distinto para el análisis del discurso que nos conecte con la práctica política. Si no hacemos eso, entonces el análisis del discurso no tendrá ningún papel positivo en algunos de los nuevos debates sobre la 'psicología crítica' que están apareciendo dentro de la disciplina.

No pretendo extenderme sobre la psicología crítica ahora, mi tarea aquí es, en cambio, considerar la cuestión del análisis del discurso dentro la disciplina. Sin embargo, debemos brevemente recordar algunas de las características centrales de la psicología que todos conocemos. Estoy preocupado aquí con el tipo de psicología anglo-americana que hoy en día es hegemónica, la cual raramente se incluye en los fenómenos que estudia, conservando su mirada dirigida a aquellos afuera de la disciplina, quienes se supone no son psicólogos y a quienes rutinariamente se manipula y distorsiona; reduce los fenómenos al nivel del individuo y en esta reducción avanza, tanto hacia abajo desde el nivel de los procesos sociales, como hacia arriba desde el nivel de las funciones fisiológicas; reproduce un modelo abstracto de las secuencias conductuales y de los mecanismos cognitivos en los cuales cada individuo se supone opera como una versión miniatura de las formas operacionales que define la investigación positivista; aparenta frecuentemente que solamente describe la actividad humana, pero esta descripción requiere de cierto grado de interpretación declarada o subrepticia, la cual prescribe la correcta versión de los hechos; y se suscribe a formas de objetividad, falsa neutralidad que oscurece el permanente rol de los intereses personales, institucionales y políticos que subyacen a la formulación de las preguntas de investigación (Parker, 2010).

No es necesario llamarse uno mismo psicólogo crítico para abogar por un enfoque que busque atender los problemas que enfrentamos en la psicología convencional a través de las siguientes preguntas: icómo se produce la psicología como un recurso sensato para que los individuos hagan un sentido de sí mismos y de los otros y de las posibilidades para el cambio de las condiciones sociales?, icómo, al nivel de la psicología, se reproducen y mantienen los procesos sociales en el rango de la interacción interpersonal y la experiencia individual?, ¿cómo están estructurados los patrones de actividad para replicar las relaciones de poder sin importar, o aun a pesar de, las intenciones inmediatas de una psicología individual?, ¿cómo puede desarrollarse la articulación teórica entre el lugar de la psicología individual y la estructura social para proporcionar alguna distancia crítica de la ideología?, icómo puede la investigación de las funciones políticas de la "psicología” operar en forma tal que mantenga un grado de autonomía de la actividad y la experiencia, de la interferencia política (Parker, 2009a)? ha habido muchos trabajando en análisis de discurso que han enfrentado estas preguntas, incluyo aquí aquellos que trabajan en el análisis conversacional, la psicología discursiva y el análisis foucaultiano. Estas preguntas son una función de la posición marginal, aunque "critica", de los analistas de discurso de los primeros años.

Sin embargo, aquí quiero destacar los problemas que aquejan al análisis de discurso en la medida en que empieza a ser aceptado como parte de la disciplina, proceso que torna el enfoque en un obstáculo más para la investigación critica, sumado a los otros problemas de la psicología que identifiqué hasta ahora. Debe ser más claro entonces por qué el giro discursivo fue un error (si bien un error necesario) y también por qué ahora necesitamos emprender otro camino. Me incluyo en este error. Podrían tomar este como el momento en que me arrepiento y les digo por qué no deben ahora tomar la ruta que una vez tome para llegar aquí. Resaltaré cinco problemas con el análisis de discurso, sugeriré algunos principios alternativos para la investigación radical de la práctica discursiva e ilustraré esto con un pequeño fragmento de texto que analizaré y contextualizaré sobre la marcha.

\section{Oscuridad}

Comencemos con el texto, primero como un texto desnudo. A medida en que se lo presento se darán cuenta inmediatamente, si han sido entrenados en la tradición del análisis conversacional del análisis del discurso, que no he marcado cada pausa, cada toma de respiración ni su articulación con los sonidos de alrededor (Potter, 1998). Esta versión fue 
grabada en 1986 y lanzada al público en 1987. La forma peculiar del texto, desde ya, levanta algunas preguntas intrigantes acerca de la trascripción, para las cuales el análisis conversacional podría ayudarnos aquí, pero dejaré de lado estas cuestiones para permanecer concentrado en temas más amplios acerca de la naturaleza del discurso y de cómo podríamos trabajar con él. En su contexto de 1986 este bloque de líneas es repetido tres veces.

Estás en negra oscuridad y confusión.

Has sido hugger-muggered y carom-shotted en una guerra.

y no sabes nada de ella.

No sabes nada de las fuerzas que la causaron, o sabes casi nada.

No vas a ganar esta guerra.

No puedes ganar esta guerra.

[El original es en inglés:

You are in black darkness and confusion.

You have been hugger-muggered and carom-shotted into a war,

and you know nothing about it.

You know nothing about the forces that caused it, or you know next to nothing.

You are not to win this war.

You cannot win this war.]

Hay algunas peculiaridades evidentes en este texto: primero, estaba planeado para una audiencia lo más grande posible, y segundo dos palabras misteriosas unidas con un guión "hugger-muggered" y "carom-shotted" que podrían leerse como repeticiones del mensaje manifiesto de que hay fuerzas de las que 'no sabes nada' o 'sabes casi nada'.

La "negra oscuridad y confusión" de la primera línea se evocan en la segunda, de tal manera que, en términos semióticos clásicos, la primera línea denota un estado físico experiencial -"tu negra oscuridad y confusión"-, y la segunda línea lo "connota" para replicar el sentido de que "tú no sabes nada" acerca de las fuerzas del lenguaje aun cuando sabes algo -que es "casi nada" en lugar de nada en sí- de manera que la líneas parecieran hacer sentido.
Un diccionario estándar ayuda con "huggermuggered" y nos dice que es una síntesis de los términos ingleses del siglo XV y XVI que se refieren a 'agruparse' y 'ocultarse'. Así, con el participio pasado del verbo tú también has sido, por estas líneas, "hugger-muggered" dentro de una visión conspirativa del mundo. La palabra "caromshotted" es más oscura; "carom", posiblemente se deriva del español 'carambola', la cual algunas veces se usa de manera figurativa para describir un truco o una trampa. En el billar una "carom-shot" describe como una bola puede ser dirigida contra otra para llevarla en cierta dirección. Así, en estas líneas tenemos una réplica inteligente de la falta de direccionalidad de la causa; somos disparados dentro de una guerra por algo que no es la primera causa; y así podemos solamente saber "casi nada" acerca de "la fuerzas que la causan". No es posible entonces ganar una guerra que está estructurada de tal manera que sus causas pueden ser evocadas pero no totalmente conocidas.

\section{Problemas del análisis de discurso}

\section{Conversación cotidiana}

Hay una creciente tendencia a enfocarse en la conversación cotidiana. Con miras a garantizar la investigación sobre los que se supone es el habla corriente, frecuentemente se estable una falsa oposición entre las entrevistas, por una parte, y la conversación que ocurre naturalmente, por la otra (e.g., Edwards \& Potter, 1992). Es decir, en una investigación a través de entrevistas todavía, al menos, tenemos la opción de ver cómo el psicólogo estructura la interacción incluyéndose en el fenómeno que está siendo estudiado (ha habido algunos muy buenos estudios de análisis conversacional dedicados a esta estructuración). El foco en la conversación cotidiana, en contraste, es cómplice con la mirada de la psicología convencional sobre la actividad de otros que se supone no son psicólogos.

En cambio del énfasis en la conversación cotidiana necesitamos (i) enfocarnos en el uso que se hace de la psicología en el dominio público y (ii) 
concentrarnos en la reproducción de las condiciones en las cuales las explicaciones psicológicas adquieren importancia. Es decir, necesitamos cuestionar de manera reflexiva las formas dominantes de conocimiento y nuestra propia inclusión en estas formas dominantes.

El texto acerca de la "negra oscuridad y confusión" sirve como un bonito ejemplo acerca de tal inclusión de posiciones de lectura, escritura e interpretación; hay razones específicas para esto que pueden ser guía de pensamiento acerca de lo que hace un discurso y de lo que podemos hacer con él.

El texto proviene de la canción "F.I.A.T." del disco titulado Opus Dei (Monroe, 2005). Estoy mostrando esto paso a paso, así podemos apreciar algunas de las capas de significado que operan aquí. El simple marco del título "F.I.A.T." es enigmático, lo cual concuerda con el sentido de que "no sabes nada de las fuerzas que la causaron o sabes casi nada', y mientras la letra de otras canciones aparecen en la cubierta de Opus Dei, la letra de esta canción no. Que esto pueda ser una referencia a la fábrica de automóviles FIAT parece descartarse cuando vamos al título del álbum en la carátula, pues si, Opus Dei significa 'Obra de Dios', entonces hace más sentido para nosotros eliminar los signos de puntuación y juntar las letras para leer "Fiat" como en "Let it Be" ('Déjalo ser'), como en las palabras de Dios "Fiat Lux", 'Que se haga la luz', o aquí también 'Que allá haya luz' en la "negra oscuridad y confusión".

El título del álbum también connota confusión y conspiración, porque el Opus Dei es conocido como una organización secreta dentro de la Iglesia Católica, la cual está también implicada en políticas fascistas. Eso nos conduce a la banda responsable de este disco, una banda que también ha sido acusada de ser fascista.

Es Laibach, cuyo nombre en sí mismo fue una provocación cuando se formó en 1980 en Trobvlie en Eslovenia, que en ese momento era la nación más al norte de la República Federal Socialista de Yugoslavia. La capital de Eslovenia es Liubliana y por eso adoptar el nombre de la ciudad en alemán, 'Laibach', fue suficiente para que se prohibiese la banda. Laibach se convirtió en una parte integral de la constelación de fuerzas políticas-culturales llamadas "El Nuevo Arte Esloveno", comúnmente conocido usando su designación alemana 'Neue Slowenische Kunst' o 'NSK'. Es posible en este momento referirse a Laibach y a NSK de manera intercambiable; miembros de NSK realizan el trabajo artístico para las carátulas de los discos y para la publicidad de Laibach (Arns, 2003).

Una de las cosas interesantes de NSK es que, insertada en su crítica explícita a la disidencia como estrategia en Europa Oriental, también hay una crítica implícita a la psicología cotidiana. El Manifiesto NSK Laibach Kunst se redactó como reacción contra los movimientos alternativos en Eslovenia y, en particular, en contra de la creencia de que la disidencia podría operar como un espacio personal en que el disidente piensa que puede estar libre de los aparatos del Estado y del partido.

El argumento era que ese espacio personal era en realidad un prerrequisito para que el régimen funcionara. El nivel de la psicología, como algo separable idealmente del contexto político, servía como una zona de amortiguación en el cual la gente podía quejarse, sentirse libre y tolerar la burocracia. El manifiesto Laibach Kunst llamaba en cambio al "principio de rechazo consciente de gustos personales, juicios, convicciones" y a la "libre despersonalización, voluntaria aceptación del rol de la ideología". Estar en "negra oscuridad y confusión" mientras se escucha el álbum Opus Dei es, por tanto, abrazar las fuerzas oscuras en lugar de intentar dominarlas, cualquier intento de aclararlas es en sí mismo visto como una treta ideológica (Cufer \& Irwin, 1992).

El papel de lo cotidiano, como algo que pareciera estar libre de fuerzas estructurantes, se pone en cuestión, y tenemos en su lugar la atención dirigida a la manera en que ciertas manifestaciones de la practica discursiva producen y reproducen formas de psicología. En contra del análisis de discurso que se enfoca en la conversación cotidiana, volvemos nuestra mirada hacia la psicología y a las fuerzas ideológicas que le dan lugar. Este primer principio necesita estar entrelazado con otros argumentos, así que movámonos a otro problema con el análisis de discurso. 


\section{Interacción interpersonal}

En la actualidad hay un interés en la interacción interpersonal. Aun cuando a menudo hay una atención explícita sobre la interacción, en vez de una búsqueda por procesos cognitivos dentro de las cabezas de los individuos participantes, este énfasis en la interacción interpersonal todavía se hace a expensas del análisis de las relaciones de poder más amplias (e.g., Edwards, 1992). Raros intentos de insertar, por ejemplo, la interacción personal en sistemas de autoridad patriarcal, necesariamente tienen que separarse de la psicología y de las formas de análisis de discurso toleradas en esta disciplina. El foco sobre la interacción personal es realmente una pieza del amplio proyecto de la psicología, el cual reduce la descripción y la explicación a la interacción de escala menor, si es posible a las operaciones mentales de los individuos involucrados.

En cambio (siguiendo la numeración de la secuencia que empecé en la sección anterior), necesitamos mostrar (iii) cómo las relaciones de poder son reiteradas en el ámbito interpersonal y (iv) cómo, ostensiblemente, procesos individuales son movilizados por cadenas más amplias de poder. Es decir, cuestionamos nociones de motivación o de intereses como artefactos explicativos, girando en su lugar hacia cómo las actuaciones mantienen las posiciones de aquellos que hablan, escuchan e interpretan.

Aquí las intervenciones político-culturales de NSK proveen un ejemplo de cómo trabajar con el discurso, de tal manera que se evite la reducción a lo psicológico o a un nivel interpersonal de explicación. El circuito de lo irracional apela a la autoridad irracional -"Fiat" insertado en Opus Dei- y el rechazo de cualquier suposición de una autoridad racional iluminada que pueda explicar convincentemente las fuerzas que llevan a la "confusión", burlar cualquier autor sapiente, hasta cualquier autor sapiente que le dirá a los que escuchan el mensaje de que esto es un juego. La insistencia en no dejar caer la máscara mantiene a este circuito mistificador andando, así que mientras que podríamos sospechar que esta es una actuación de alguna clase Laibach no nos dirá que es una actuación excepto en el sentido de que "sabemos nada" o "casi nada".

Un aspecto perturbador de las presentaciones de Laibach durante los años 80 , cuando este texto fue lanzado, y en los años 90 después de la desintegración del Estado Yugoslavo, fue que la banda siempre aparecía en público en uniforme. Los miembros de la banda nunca se quitaban la máscara para asegurar a la audiencia de que solo en apariencia eran fascistas, pero que en realidad no lo eran (Žižek, 1993a). Esta clase de actuación desbarataba así el intento de anclar y explicar la presentación por la vía de la intención individual, y en su lugar pone un corto circuito entre, el nivel de la actuación, cuando la banda le dice a la audiencia que ellos están en "negra oscuridad y confusión", y los más altos niveles del Estado yugoslavo cuya autoridad replicaban y así socavaban. El mensaje de que "tú no puedes ganar esta guerra" se lanzó cuando los conflictos entre diferentes grupos nacionales en el Estado yugoslavo estaban empezando a tornarse violentos; este es el año en que Slobodan Milošević visitó Kosovo y allá declaró su apoyo a las aspiraciones nacionalistas serbias (Jeffs, 1995; Magaš, 1993).

Dentro de Eslovenia, ese año, 1987, fue cuando surgió el 'escándalo del afiche' que terminó con el Día de la Juventud Yugoslava, el cual era no coincidencialmente el día del cumpleaños de Tito. Un afiche enviado por el grupo de diseño NSK Nuevo Colectivismo ganó la competencia al mejor trabajo, pero después de que el panel de jueces había declarado que el diseño ganador encarnaba el espíritu de la juventud socialista yugoslava, se reveló que este estaba basado en un afiche de propaganda Nazi de 1936 (Stepančič, 1994).

Así que en vez de optar por la disidencia, el NSK había abrazado la ideología y lo había hecho de manera tan efectiva que la desmanteló desde adentro. Ninguna apelación a lo que en realidad quería decir el autor fue necesaria, y en su lugar las formas de representación y su función en los regímenes simbólicos de poder históricamente-constituidos fueron volcadas contra sí mismos.

Aquí, el nivel de lo interpersonal no se privilegia sobre otros niveles de práctica y una reducción al nivel de los procesos mentales individuales es cui- 
dadosamente dejada de lado. Contra el análisis de discurso que continúa enfocándose sobre la interacción interpersonal tenemos, entonces, una manera de trabajar con la práctica discursiva que conecta con el nivel de juicio personal en una forma que lo cuestiona. Formas de representación son tratadas como sitios para el ejercicio del poder y así hay una manera muy distinta de localizar los lugares de intervención que interpretan y mantienen o cambian las condiciones sociales. Este es el segundo principio de una aproximación alternativa a la práctica discursiva. Continuemos para ver cómo podemos llegar al tercero.

\section{Secuencias formales}

Existe un énfasis en el análisis de discurso ahora en las secuencias formales de interacción. Estas secuencias formales avanzan independientemente de cualquier contenido particular, esto significa a su vez que el contexto de significación es usualmente eliminado del análisis como parte de un intento para evitar una referencia al significado real, propuesto por los actores sociales (e. g., Antaki, 2008). Esta también es la racionalidad para evitar complicaciones éticas que pudieran surgir si las interpretaciones se les devuelven a los participantes, quienes podrían no estar de acuerdo con lo que dice el analista de discurso acerca de lo que ellos han dicho. Este énfasis en la forma a expensas del contenido repite el esfuerzo de la así llamada psicología científica, al remplazar el significado asignado por la gente a las situaciones con re-descripciones mecanicistas y frecuentemente deshumanizadoras acerca de su conducta y sus mecanismos cognitivos ocultos.

En cambio, necesitamos mostrar (v) cómo los dispositivos formales son inyectados con un contenido particular y (vi) cómo los dispositivos formales son re-significadas en distintos contextos sociales. De esta manera, hacemos patente cómo las formas simbólicas operan como parte del proceso histórico y cómo los dispositivos formales cargan el peso de la historia, repitiendo y transformando las condiciones sociales.

Arrancar las imágenes políticas de su contexto fue una de las características del punk en occiden- te, y su aparición en Eslovenia impulsó el movimiento de oposición en la última parte de los años 70. Estrategias de "détournement" usadas por los situacionistas fueron influyentes y también fueron usadas por NSK (Spanke, 1993). Al titular la canción "F.I.A.T.", por ejemplo hay una anticipación del álbum de Laibach lanzado al año siguiente en el cual hay una réplica y una re-significación del álbum de los Beatles "Let it Be". En la versión de Laibach de "Let it Be" cada canción del álbum original es re-presentada, exceptuando a la canción de la portada "Let it Be" (Monroe, 2005). Así, por ejemplo, la primera canción "Get Back" incluye las letras originales "Get back to where you once belonged" ('regresa al lugar al cual alguna vez perteneciste'), pero al entonarla de manera amenazante por hombres en uniforme las implicaciones fascistas latentes son traídas a la superficie. De igual manera, en Opus Dei, la canción de Queen "One Vision" ('Una Visión') incluye la letra original que invita a una celebración frenética de una nación, pero que ahora en alemán en la canción de Laibach 'Gesburt Eiener Nation' (un título que hace eco del protofascismo de la película "el nacimiento de la nación" con la cual en 1915 se celebró la formación del Ku Klux Klan en los Estados Unidos).

Hay otro proceso crucialmente importante de re-significación trabajando en la canción de 'F.I.A.T.', uno que ahora sale a la luz si alguna de esas frases peculiares se escribe en los buscadores de Internet. Resulta que hay una conexión entre los caracteres iniciales que deletrean "Let it Be" y la industria automotriz; la FIAT opera en Italia vecina a Eslovenia, antiguo poder invasor bajo Mussolini, entonces aparecen otras reivindicaciones históricas por el territorio hechas por nacionalistas tanto en el lado italiano como esloveno.

El bloque de líneas se re-significa de 1942, en Italia, a un periodo de agitación y guerra inminente en Yugoslavia en 1980. Pues fue el 13 de julio de 1942 cuando estas palabras fueron compuestas por primera vez y transmitidas por Radio Roma a las fuerzas armadas de Estado Unidos que entraban a la guerra en Europa. La transmisión radiofónica es "Darkness" ('oscuridad') de Ezra Pound; así, el mensaje a los norteamericanos es que ellos han sido 
'hugger-muggered' y 'carom-shotted' a una guerra de la que "no saben nada acerca de las fuerzas que la causaron" o "casi nada" (Tiffany, 1990). El reemplazo de las líneas en su contexto original tiene el efecto de llenarlas con un significado diferente, pero mientras esto parece proveer una explicación del ahora carácter más bien arcaico y bizarro del texto, esta re-significación también entonces pone en cuestión la intencionalidad del autor de las líneas replicadas como una canción en un álbum de Laibach.

NSK muestra su propio brillo teórico sobre esta actividad de re-significación en el cual un principio "retroactivo" en la producción del arte es anclado, al parecer, por el uso de dispositivos puramente formales. Uno de los puntos de referencia claves es el movimiento de arte abstracto "suprematismo" fundado por Casimir Malevich en Rusia antes de la Revolución, el cual fue incorporado como un recurso de vanguardia, agit-prop en el fermento creativo de los primeros años de la Unión Soviética (Mudrak, 2001). Conocido por el "Círculo Negro" el "Cuadrado Negro" y la "Cruz Negra”, los cuales aparecen una y otra vez en los proyectos de arte de NSK y como material simbólico sobre los uniformes de Laibach. Malevich en sí es re-significado. Estos son los motivos perfectos para evocar la "negra oscuridad" y "confusión". Malevich nunca fue un bolchevique, pero se lo representó como si fuera uno, ni siquiera ruso, sino reclamado por Ucrania. NSK se basó en estos recursos en conjunto con otros elementos realistas de la sociedad soviética y de la iconografía del nacional socialismo alemán para construir un auténtico arte esloveno (Gržnic, 2004).

Aquí hay un giro de NSK hacia las propiedades formales de la representación, pero precisamente para romper la reducción a tales formas. Contra el análisis de discurso que bien intenta reducir la actividad significativa a secuencias formales, y en contra de las actividades fenomenológicas que privilegian el contenido como una reacción opuesta a tal reducción a la forma, podemos ver como las prácticas discursivas constituyen y reconstituyen formas simbólicas. Así, tenemos un tercer principio ahora elaborado en una aproximación muy diferente al discurso en el cual trazamos como las formas sociales de manera situadas contextual e históricamente movilizan contradictorios y competentes contenidos ideológicos. Movámonos hacia un cuarto problema con el análisis de discurso en psicología.

\section{Explicación correcta}

Hay un foco sobre la correcta explicación del habla. A pesar de la invocación repetida de principios etno-metodológicos concernientes al propio raciocinio de las personas, el análisis conversacional procede por medio de una detallada re-descripción del habla usando un vocabulario especializado que traduce una trascripción complicada de interacción (e.g., Atkinson \& Heritage, 1984). Otras formas de análisis de discurso que intentan descubrir discursos o repertorios proceden en la misma forma, reemplazando el texto actual con una re-descripción teórica que a su vez funciona como una interpretación. Esto es, por mucho, una conservación de la reinterpretación psicológica de la conducta en términos de mecanismos cognitivos o la parafernalia en algún otro vocabulario particular. En cambio, necesitamos (vii) aprender de la manera en que las interpretaciones y las reinterpretaciones en realidad existentes combaten versiones de la realidad y (viii) prestar atención a los espacios conflictivos en los cuales hay una lucha por la naturaleza de la correcta explicación. Esto significa trabajar con prácticas discursivas antagonistas y abiertas que cuestionan cualquier sistema ideológico de significaciones cerrado consensualmente.

Así, podemos ver en el caso del NSK que la "aceptación voluntaria del papel de la ideología" es una estrategia de inmersión en el discurso que evita la trampa ideológica de imaginar que podemos comentar sobre el discurso desde una posición que es completamente independiente de este. Para el NSK, es la idea de que uno puede hablar afuera de los sistemas ideológicos lo que sostiene la posición disidente en Europa Oriental, así como la idea de un espacio personal libre es también la que consuela a los consumidores y audiencias de la propaganda política en el mundo capitalista. Además, la posición desde la cual se habla con uno acerca de las fuerzas de las cuales sabemos "casi nada" no se le 
atribuye ningún conocimiento superior que el que tenemos nosotros mismos (Žižek, 1994).

Mis informantes NSK me dicen que el mensaje del programa de Ezra Pound "Darkness" no estaba dirigido solamente a Pound, quien estaba atrapado en la "negra oscuridad y confusión" del fascismo italiano y en la locura después de su captura por los norteamericanos. Fue un mensaje que también estaba dirigido hacia sí mismos, NSK, un recordatorio de que el artista no tiene necesariamente una posición privilegiada para comentar sobre los asuntos políticos en los que interviene.

La nacionalidad y el Estado proveen anclas para el sí-mismo, ellos imitan y reproducen las afirmaciones ideológicas de que hay una identidad auténtica que puede ser puesta en contra las fuerzas externas. Un Estado Esloveno aparentemente independiente fue la primera nación en ser sacada de la federación yugoslava por Occidente en 1991, el NSK, que había estado ensamblando su propia versión de la autentica identidad eslovena muy deliberadamente ensamblada desde fuera de la nación, respondió erigiendo su propio Estado, el Estado NSK en el tiempo.

La embajada del NSK en Sarajevo en 1995 distribuyó pasaportes diplomáticos que eran tan convincentes que algunas personas lograron salir de Bosnia-Herzegovina durante la guerra civil. Laibach, que tocó en Sarajevo en su "Gira de Europa Ocupada", jugó un papel específico como políticos en el Estado en el Tiempo NSK. Hay artistas del Estado (el Grupo Irwin) y una Iglesia Estado (Teatro Cosmokinético Noordung) y junto con el grupo de diseño Nuevo Colectivismo, este grupo de organizaciones político culturales proveen a través de su propio aparato de Estado ciudadanía para quien aplique sin importar el territorio geográfico (Arns, 1998).

Este Estado es un Estado en el Tiempo, no en algún territorio particular, así desmantela nuestras suposiciones acerca de la filiación a una identidad que es organizada alrededor de un centro, la clase de identidad valorada bajo el capitalismo. Hay además, por tanto, un desmantelamiento del aparato del Estado del yo, es decir, de la disciplina de la psicología. También debe notarse que el NSK Estado en el tiempo, no es democrático y no pretende ser un espacio abierto consensual en el cual todos los ciudadanos participan libremente.

Hay proyectos en curso para acumular experiencias históricas de aquellos con pasaportes NSK; el grupo Irwin está actualmente trabajando con los relatos de nigerianos portadores del pasaporte NSK. Hay proyectos continuos para acumular las experiencias históricas de aquellos con pasaportes NSK, por ejemplo el grupo Irwin actualmente está trabajando con testimonios de nigerianos portadores del pasaporte. Sin embargo, a manera de ilustración las oficinas de pasaporte están estructuradas deliberadamente para replicar el aparato de Estado y para repetir y así cuestionar la naturaleza autoritaria de todas las modalidades de Estado y de identidad (Žižek, 1993b).

Insistir sobre el carácter antagónico, no-consensuado del discurso pone en cuestión la supuesta independencia de los investigadores académicos y su confortable suposición de que ellos están en una posición para explicar lo que otros quieren decir. Contra el análisis de discurso que pretende proveer explicaciones correctas, entonces, resaltamos formas de práctica discursiva que abre espacios para la interpretación y argumentamos acerca de la naturaleza de la interpretación.

\section{Segregación disciplinaria}

Ahora atendamos al quinto problema con el análisis del discurso, que se refiere a la evitación deliberada de la política. Esta evitación va desde la solicitud de que el investigador no hable del poder en su análisis de un texto si el tema del poder no es tratado allí, hasta la estrategia retórica de desviar preguntas políticas a través de insistir que estas no son parte de la investigación y que pueden ser consideradas en otro departamento académico (e. g., Hepburn \& Wiggins, 2007). Esta especialización sub-disciplinaria en discurso, que limita este dominio de otras áreas de trabajo se adecua muy bien en los confines de la segregación disciplinaria que los psicólogos desean conservar en su lugar, es también el lugar para pretender la neutralidad de la investigación psicológica. 
En cambio, necesitamos mostrar (ix) cómo la política enmarca el trabajo psicológico y $(\mathrm{x})$ las maneras en que el proceso de psicologización en la sociedad contemporánea es profundamente política. Esto significa, aparentemente, conectar respuestas afectivas individuales al material simbólico con procesos políticos.

Esto aquí origina preguntas particulares acerca del llamado del texto de "F.I.A.T." y de la manera en que un tipo de respuesta del lector se construye haciendo eco a las ambigüedades e incertidumbres a nivel de lo político. Hemos notado como el uso de un giro particular de una frase -"huggermuggered" y "carom-shotted"- evoca, como parte del tono del mensaje, la "confusión" que describe. La formulación peculiar 'no sabes casi nada' acerca de las fuerzas que causaron esta guerra tiene un lado siniestro en sí mismo porque elicita el sentido que como individuos tenemos indicios de que lo que sabemos puede ser verdad pero en lo que no podemos confiar, y el sentido de que hay fuerzas políticas que nos llevan a hacer cosas acerca de las cuales tenemos sospechas confusas e incompletas.

No sorprende que una transmisión fascista deba insinuar conspiraciones, pero el oficio retórico de Pound conecta la conspiración global con el sentido que todos los individuos alienados tienen de aquello que no saben que yace dentro de ellos mismos. Una versión de la psicología individual es así parte de la fuerza política que Pound transmite, posteriormente esta es insertada en otro marco político por Laibach. Como una contraparte de la transmisión de Pound, el álbum Opus Dei incluye otra reciente canción "the great seal" ('el gran sello'), el cual es una marcha pomposa y lenta con las palabras de Wiston Churchill, el discurso que declara que "los combatiremos en las playas".

La música y las letras están reelaboradas en el álbum de Laibach de 2006, Volk, como el himno nacional de NSK al que se ha añadido las palabras "combatiremos para defender nuestro Estado". El disco Volk está compuesto por himnos nacionales que han sido variados, algunos con muy pocos cambios de las letras originales pero re-significados a través del uso de ritmos distintos y, más recientemente, en algunos casos, con videos acompañantes.
La canción "Anglia”, por ejemplo, tiene versos burlones referentes a los ingleses imaginando que aún rigen el mundo, que "todas las naciones son inferiores, cualquier alzamiento silenciado, los escoceses rebeldes destrozados". Las referencias a destrozar a los escoceses en realidad continúan en el poco cantado cuarto verso del verdadero himno nacional inglés. En la versión de Laibach el coro concluye con "Dios salve a su gracia la reina, Dios los salve a todos". El video tiene la figura de una señora mayor vestida en bata, alimentando a la fuerza con un desayuno inglés a prisioneros esposados. La canción española proclama que España es un fantasma de ETA. El tour de Estados Unidos se tituló Los Estados Divididos de América, con el lema publicitario 'Unidos Caeremos'. Estos videos se pueden encontrar fácilmente en la sección Laibach de nskstate.com de la página web y, por supuesto, en YouTube.

Símbolos políticos son así desplegados por NSK para traer a la superficie las siempre presentes funciones y repercusiones políticas del discurso. Mientras el Estado yugoslavo se desintegraba al final de los ochenta e intentaba salirse de la guerra civil que estaba envolviendo a Croacia, Serbia y Bosnia-Herzegovina, NSK intervino en una manera muy deliberada para agudizar la tensión. Afiches callejeros fueron colocados por el grupo Nuevo Colectivismo NSK, el mismo grupo que había estado detrás del Escándalo del afiche en 1987. Los afiches hacían explicito el supuesto que circulaba en Eslovenia de que el mercado libre resolvería sus problemas. Un afiche declaraba "iCompra la victoria!". Otro afiche, con palabras que estaban en croata en vez de esloveno, salió a las calles de Liubliana con el lema: "iquiero pelear por una nueva Europa!” (Monroe, 2005). El papel de la política en la vida cotidiana es así traído a la luz, hay un rechazo a segregar las política, a tratarla como si fuera algo que solo concierne a los políticos o como algo concerniente a fuerzas de las "cuales no sabemos nada" o "casi nada".

Contra el análisis de discurso que intenta estudiar el lenguaje y al sí-mismo sin referencia a contextos sociales e históricos más amplios y que hace el mismo juego que la psicología que excluye las preguntas políticas, tenemos un quinto principio 
para la práctica discursiva que NSK representa, esto es, necesitamos conectar el reino de contradictorias fuerzas afectivas individuales con el reino de la lucha política.

\section{Conclusiones}

Sería tentador, especialmente a la luz del título de la canción "F.I.A.T." decir que debemos girar hacia algo que podría ser llamado investigación "analítica auto- discursiva", pero esto podría poner en riesgo la práctica discursiva de NSK, asimilándola a algo que es espontáneo. Los analistas de discurso en psicología desarrollaron sus trabajos desde marcos teóricos muy específicos, aun cuando algunas veces, hoy en día, pretendan que están simplemente haciendo buena investigación empírica; también las clases de análisis de discurso que he descrito están inmersas en complejas tradiciones teóricas. Tenemos que aprender que nuestra relación con prácticas discursivas teóricamente informadas tendrá que ser bastante distinta de aquella de investigadores académicos que pretenden encontrar cosas que las otras personas no sabían de antemano. Esta crítica al análisis del discurso abarca preguntas acerca de cuál debería ser nuestro papel como aliados, partidarios y voces de los análisis de discurso que se llevan a cabo fuera de la disciplina. Cuando leemos un texto acerca de la guerra de 1942 que es resignificado en 1986, también enfrentamos algunas preguntas acerca de las consecuencias que pueden traer el re-significar este texto de nuevo en una Europa al borde de la guerra.

NSK es hoy una de las muchas "micro-naciones" que juguetonamente subvierten las pretensiones de los Estados nacionales de definir y mantener las identidades individuales de sus sujetos. Tácticamente, NSK funciona como una "micro-nación", pero en realidad es mucho más como una "macro-nación”, de-construyendo naciones, de-construyendo micro-naciones del sí-mismo.

Algunas de estas micro-naciones son formadas por individuos que van esculpiendo su propio territorio o son erigidas por organizaciones de caridad que explícitamente cuestionan nociones de ciudadanía internacional en tiempos de migración masiva (Fussell, 2004). NSK y el NSK Estado en el Tiempo, NSK sin embargo, levanta las apuestas políticas para crear espacios discursivos antagónicos, re-significando formas ideológicas existentes para mostrar cómo las relaciones de poder son repetidas en cada nivel de lo social y así combaten las condiciones que hacen posible aparatos disciplinarios como la psicología (Parker, 2005, 2007a, 2007b, 2009b). Se muestra así una manera alternativa de trabajar con la práctica discursiva que también necesariamente está contra el análisis de discurso en la psicología de hoy.

\section{Referencias}

Achcar, G. (2006). The clash of Barbarisms: The making of the New World disorder. London: Saqi Books.

Agamben, G. (1998). Homo sacer: Sovereign power and bare life. Stanford: Stanford University Press.

Arns, I. (1998). Mobile states/shifting borders/moving entities: The Slovenian artist's collective Neue Slowenische Kunst (NSK). En I. Arns (Ed.), Irwin: Retroprincip 1983-2003. Frankfurt: Revolver.

Arns, I. (Ed.). (2003). Irwin: Retroprincip 198-2003. Frankfurt: Revolver.

Antaki, C. (2008). Formulations in psychotherapy. En A. Peräkylä, C. Antaki, S. Vehviläinen \& I. Leudar (Eds.), Conversation analysis and psychotherapy (pp. 26-42). Cambridge: Cambridge University Press.

Atkinson, J. M. \& Heritage, J. (Eds.). (1984). Structures of social action: Studies in conversation analysis. Cambridge: Cambridge University Press.

Baudrillard, J. (1995). The Gulf war did not take place. Bloomington: Indiana University Press.

Blackburn, S. (2005). Truth: A guide for the perplexed. Harmondsworth, UK: Penguin.

Cufer, E. \& Irwin (1992). NSK State in Time. En I. Arns (Ed.), Irwin: Retroprincip 1983-2003. Frankfurt: Revolver.

Edwards, D. (1992). Discourse and cognition. London: Sage.

Edwards, D. \& Potter, J. (1992). Discursive psychology. London: Sage.

Fussell, J. (2004). The Izbrisani (erased residents) issue in Slovenia. Recuperado el 9 de marzo, 2006, de http://www.preventgenocide.org/europe/slovenia/ 
Gržnic, M. (2004). Situated contemporary art practices: Art, theory and activism from (the east of) Europe. Ljubljana: Založba ZRC.

Magaš, B. (1993). The destruction of Yugoslavia: Tracking the break-up 1980-92. London: Verso.

Hepburn, A. \& Wiggins, S. (2007). Discursive research in practice: New approaches to psychology and interaction. Cambridge: Cambridge University Press.

Jeffs, N. (1995). Transnational dialogue in times of war: The peace movement in ex-Yugoslavia. Radical Philosophy, 73, 2-4.

Monroe, A. (2005). Interrogation machine: Laibach and the NSK State. Cambridge, MA: MIT Press.

Mudrak, M. (2001). Neue Slowenische Kunst and the Semiotics of Suprematism. En I. Arns (Ed.), Irwin: Retroprincip 1983-2003. Frankfurt: Revolver.

Parker, I. (2002). Critical discursive psychology. London: Palgrave Macmillan.

Parker, I. (2004). Discursive practice: Analysis, context and action in critical research. International Journal of Critical Psychology, 10, 150-173.

Parker, I. (2005). Laibach and enjoy: Slovenian theory and practice. Psychoanalysis, Culture \& Society, 10(1), 105-112.

Parker, I. (2007a). The truth about overidentification. En P. Bowman \& R. Stamp (Eds.), The truth of Žižek (pp. 144-160). New York: Continuum.

Parker, I. (2007b). Analfabetismo emocional: márgenes de la resistencia (E. Pascale, Trad.). En Círculo de Bellas Artes (Comp.), La fragilización de las relaciones sociales (pp. 159-187). Madrid: Círculo de Bellas Artes.
Parker, I. (2009a). Psicología crítica: ¿Qué es y qué no es? Revista Venezolana de Psicología Clínica Comunitaria, 8, 139-159.

Parker, I. (2009b). Marxismo, Psicanálise e o Estado: lições da Eslovênia. A Peste: Revista de Psicanálise e Sociedade e Filosofia, 1(2), 293-312.

Parker, I. (2010). La psicología como ideología: contra la disciplina. Madrid: Catarata.

Potter, J. (1998). Fragments in the realization of relativism. En I. Parker (Ed.), Social constructionism, discourse and realism (pp. 27-45). London: Sage.

Spanke, D. (2003). Irwin doesn't believe in Deer: Irwin's icons, kitsch, propaganda, and art'. En I. Arns (Ed.), Irwin: Retroprincip 1983-2003. Frankfurt: Revolver.

Stepančič, L. (1994). The poster scandal: New Collectivism and the 1987 Youth Day. En I. Arns (Ed.), Irwin: Retroprincip 1983-2003. Frankfurt: Revolver.

Tiffany, D. (1990). Phantom transmissions: The radio broadcasts of Ezra Pound. SubStance, 19(1), 53-74.

Žižek, S. (1993a). Why are Laibach and NSK not fascists? En I. Arns (Ed.), Irwin: Retroprincip $1983-$ 2003. Frankfurt: Revolver. Recuperado el 8 de noviembre de 2005, de http://www.nskstate.com/

Žižek, S. (1993b). Es gibt keinen Staat in Europa. En I. Arns (Ed.), Irwin: Retroprincip 1983-2003. Frankfurt: Revolver. Recuperado el 3 de agosto de 2003, de http://www.nettime.org/desk-mirror/zkp2/staat. html

Žižek, S. (1994). The enlightenment in Laibach. In I. Arns (Ed.), Irwin: Retroprincip 1983-2003. Frankfurt: Revolver. 